\title{
JAVA REAL-TIME PUBLISH-SUBSCRIBE MIDDLEWARE FOR DISTRIBUTED EMBEDDED SYSTEMS
}

\author{
Daeyoung Kim, Yoonmee Doh, \\ Yann-Hang Lee* \\ \{dkim,ydoh\}@cise.ufl.edu \\ University of Florida, Gainesville, FL, USA \\ *yhlee@asu.edu \\ Arizona State University, Tempe, AZ, USA
}

\begin{abstract}
As the complexity of distributed embedded systems is increasing, and frequent upgrades and maintenance are expected, conventional communication models, such as point-to-point and the client-server model are not adequate to keep up with these trends. So we propose a generic Java real-time communication model based on the publish-subscribe model which has proper characteristics for distributed applications. The proposed Java publish-subscribe model has been implemented as middleware, named Java Embedded Bus (JEB). To easily support heterogeneous communication technologies, we propose $a$ middleware-independent plug-and-play JEB device driver model. We have also prototyped the middleware on the demonstrative distributed embedded systems which comprised embedded machines and workstations connected by VME bus and Ethernet.
\end{abstract}

\section{Introduction}

Java network computing technology has had a significant impact on distributed systems that are typically connected by TCP/IP Internet. Since distributed systems are composed of heterogeneous computing systems, i.e. different hardware and operating systems, Java's compatibility of byte codes and unified communication interface have tremendous appeal in the area of distributed systems. Inspired by the success of Java in conventional systems, there are significant efforts to adopt Java in embedded systems. The Java technology has already shown its success in Internet appliances such as set-top box, PDA, and hand-held-computers. Therefore, it is strongly anticipated that Java will be a major programming environment in complex distributed embedded systems in the near future.

The original version of this chapter was revised: The copyright line was incorrect. This has been corrected. The Erratum to this chapter is available at DOI: 10.1007/978-0-387-35409-5_23 
The reasons why Java is favorable in distributed embedded systems are as follows. Firstly, Java provides a reliable and secure computing environment, ensured by Java's safety features such as strong compile- and run- time verifications, an efficient exception-handling-mechanism and sand-box security model. Secondly, Java provides strong portability and reusability, which industry can take advantage of, reducing costs and development turn around time. Despite its versatile advantages, Java still has weaknesses in applications of embedded systems that require strict timing constraints and deterministic behaviors. For example, Java's problems are undeterministic behavior of garbage collection, lack of direct hardware access, low performance, and weakness of synchronization, etc. However, with the early consensus of these problems in both industry and academia, there is ongoing work for the standardization of real time Java specification.

Current real time Java working groups, Real-Time Java Experts Group and JConsortium, focus only on solving fundamental weaknesses of Java in real-time applications as mentioned above. Their objectives do not include practical communication middleware architecture for distributed embedded systems. Unlike conventional distributed systems, distributed embedded systems are connected by a variety of communication technologies such as VME bus, FieldBus, IEEE P1394, ARINC, and FDDI, as well as legacy Ethernet. In such cases, we can not use Java's popular communication model, TCP/IP over Internet, without expensive solutions. Also, Java's communication model does not consider real-time constraints.

In this paper, we propose Java publish-subscribe middleware that provides communication facilities for distributed embedded systems that are connected by heterogeneous communication technologies. As the complexity of distributed embedded systems increases, and more frequent upgrades and maintenance are expected, conventional communication models such as point-to-point and clientserver model are not adequate to keep up with these trends. So we have built generic Java communication facilities based on the publish-subscribe model which has become popular in the area of distributed communication, due to its easy adaptation to system evolution and scalability. We have also added real-time specific features to the publish-subscribe model to guarantee the timing requirements of distributed embedded systems.

The proposed Java publish-subscribe model has been implemented as middleware, named Java Embedded Bus (JEB), between the Java virtual machine and Java applications. To easily support heterogeneous communication technologies, we developed a middleware-independent plug-and-play JEB device driver model. The prototype middleware, JEB, is being built on the demonstrative distributed embedded system which comprises PowerPC embedded machines and IBM PCs that are connected by VME bus and Ethernet. The prototype platforms also include VxWorks, Linux, Sun's JVM, and Personal JWorks.

The rest of the paper is structured as follows. We describe the design goals in section 2. The generic Java communication model is discussed in section 3 . We show the details of the middleware in section 4. A short conclusion then follows.

\section{Design Goals}


Our main objective is to purely extend the Java language system for building a distributed Java embedded application environment. The detailed design goals are to support followings:

Generic Real Time Communication Model - there are a variety of communication requirements in practical distributed embedded systems. They are relevant to the complexity and communication behaviors of applications. In simple cases, the point-to-point communication model is enough. But, as for safety critical battleship control systems that require redundancy based fault tolerance, a faulttolerance supporting communication model is necessary. The model should be powerful enough to support these various communication requirements.

Adherence to Java Programming Style - one of the advantages of using Java is its simplicity of programming style with the help of a simple language structure and an object oriented paradigm. Therefore, the best approach for Java communication is to exchange serializable Java objects themselves. The communication middleware should cause a minimum impact on the conventional way of Java programming.

Real Time Behaviors -Java's TCP/IP communication model cannot guarantee real-time constraints such as message deadline, jitters, etc. In order to guarantee realtime constraints of distributed embedded systems, real-time control functions must be provided in the middleware level.

Middleware Independent Plug-and-Play Device Driver Model - to provide the same interface to application programmers for different communication technologies, a middleware independent plug-and-play device driver model is required. Following this model, application programmers can significantly reduce their efforts while changing their hardware dependent communication part.

Scalability and Interoperability - the model should be scalable upon the growth of the communication infrastructure. Interoperability among heterogeneous communication technology is also important.

Multicast/Broadcast Support - since a publish-subscribe model has characteristics in common with multicast/broadcast protocol by inheritance, it is advised to take advantage of lower level support of multicast/broadcast protocol while integrating with lower level communication technology.

\section{Java Publish-Subscribe Real-Time Communication Model}

The building of a Java real-time communication model should begin with analyzing communication requirements of real distributed embedded systems. In this section, we have classified communication requirements of distributed embedded systems and have built a Java publish-subscribe real-time communication model which fulfills the requirements.

\subsection{Generic Communication Requirement}

We have classified communication requirements of distributed embedded systems into categories of connection types, connection modes, and data types.

The connection types of distributed embedded systems are categorized into the four types, according to their relationship between source and destination. They are 
point-to-point, point-to-multipoint, multipoint-to-point, and multipoint-tomultipoint. The latter two types are very important when we build replication-based fault tolerant distributed embedded systems. In fault tolerant system, an application is usually duplicated in different processors to cope with a failure of the master application.

According to the behavior of a thread (or process) after issuing a send or receive primitive, we can distinguish the mode of a connection; either asynchronous or synchronous connection. After a sender transmits an asynchronous message, it does not wait for either an acknowledgement or a response from the receiver. So the sender does not block itself after transmitting an asynchronous message. For a synchronous message, a sender has to be blocked after transmission until it receives either an acknowledgement or result.

According to the semantics of messages to be exchanged, we can classify three data types, signals, commands/requests, and events. Signals are defined as unidirectional data flows that carry continuous information such as sensor data. Signal data communication is typically characterized as time-critical, updates are useless if old, idempotent, repeated updates are acceptable, last-is-best, latest information is more important than retrying missed samples. Regarding commands/requests, application cannot miss any intermediate commands or execute a command twice. Similarly embedded applications occasionally need to issue specific requests for data. Commands/Requests imply a two-way transaction. Events are used to synchronize task execution with asynchronous operations. For example, pump tasks do not run until the float task indicates the level has fallen below a set value.

\subsection{Real Time Java Publish-Subscribe Model}

Some of the most important objectives while building distributed embedded systems are simplicity, evolvability, scalability, and reusability. The conventional clientserver model is not well suited to these purposes. For example, when the complexity of a system is high, there may be no clear decomposition of servers and clients. Also dynamic or static addition or removal of applications will be a very difficult job.

The publish-subscribe model is a very good candidate to overcome these problems. In the publish-subscribe model, a sender does not need to know who needs its message and a receiver does not care who sends the message. This scheme greatly reduces the complexity of communication architecture and makes it easy to upgrade and reuse the application components. Therefore, we have adopted a publishsubscribe model as a core architecture of our middleware.

3.2.1. Basic Publish-Subscribe Architecture. Basically, all threads (processes) which need to communicate with others must join the publish-subscribe group. Then, a publisher which wants to publish its messages has to register its message subject to the publish-subscribe group. The subscriber which needs the message should register itself to the specific subject group. When a subscriber registers the subject, the subscriber is enrolled in the publisher's destination list. So whenever the publisher publishes data, it will be forwarded to the subscriber. The described basic publishsubscribe architecture is shown in Figure 1. 


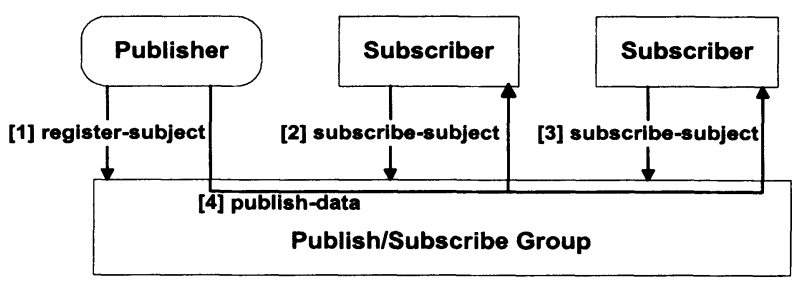

Figure 1. Basic Publish-Subscribe Architecture

3.2.2. Enhanced Publish-Subscribe Architecture. To meet the requirements of distributed embedded systems, we have enhanced the basic publish-subscribe model by adding several new features as follows.

3.2.2.1. Real-time support. There have been significant research efforts to guarantee real-time constraints in priority based scheduling. To take advantage of these research works, we also provide a priority based scheduler for publishers and subscribers. For a publish-subscribe subject, we allow priorities to be set along the route of a message. For example, in a publisher's processor environment, the message will be scheduled based on publisher-priority. On the subscriber's side, an incoming message will be scheduled based on its subscriber-priority. In order to enforce the negotiated traffic behavior, the middleware is also equipped with a traffic shaping function.

3.2.2.2. Synchronous communication support. Basic publish-subscribe architecture is built on an asynchronous communication model. So the message flow between a publisher and a subscriber is uni-directional. But data types such as commands/requests require a two-way communication, and a publisher should be blocked until it gets either an acknowledgement or a response from the subscriber. When it registers a subject in the publish-subscribe group, two corresponding subjects are enlisted to accommodate a two-way communication. In this case, the middleware should support blocking the current publisher after publishing a message until a message is received from the subscriber.

3.2.2.3. Fault tolerance support. In multipoint-to-point and multipoint-to-multipoint, we provide two different semantics for each publisher and subscriber, to support diversity of real time applications. In combination, we support four different semantics for these two connectivities. Firstly, on the publisher side, multiple publishers can be either in master/shadow model or client-server model. In the master/shadow model, only one publisher can be an active publisher whose data can arrive to registered subscribers. The data from other publishers, shadow publishers, are forced not to send to subscribers. This model is very useful in replication based fault tolerant systems. Only the active copy of the application can send the data. The election scheme such as primary-backup and leader elections are supplied in the middleware level to alleviate the burden of programmers.

3.2.2.4. Reservation based rate control support. We also support a rate adjustment capability to guarantee timing requirements of both publisher and subscriber. For example, the publisher registers that it can publish sensor data at a frequency of $1000 \mathrm{~Hz}$. And two subscribers need the sensor data at a frequency of $1000 \mathrm{~Hz}$ and $10 \mathrm{~Hz}$ respectively. In this case, the publisher can not be allowed to publish sensor data at $1000 \mathrm{~Hz}$ for both subscribers. The subscriber which expects $10 \mathrm{~Hz}$ sensor data 
may fail due to too many arrivals of sensor data. The middleware provides subscriber initiated negotiation protocol and guarantees the negotiated rate.

\section{Java Real-Time Communication Middleware}

We have implemented the Java publish-subscribe real-time communication model as middleware, which is located between the Java virtual machine and Java applications. Since the logical behavior of the publish-subscribe model is similar to that of a hardware bus, we named the middleware Java Embedded Bus (JEB). The JEB is a three-layer architecture, which includes JEB API, JEB Family, and JEB device driver from top to bottom. We show the general architecture of the JEB in Figure 2. In this section, we describe JEB Family, JEB API and JEB device driver.

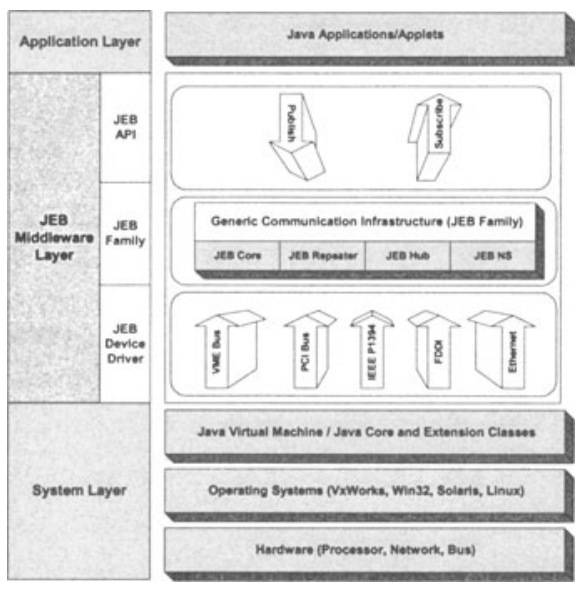

Figure 2. JEB Middleware Architecture

\subsection{JEB Family}

JEB Family is the core part of the middleware, and provides distributed Java applications with real time communication facilities such as routing, control and maintenance, and real time scheduling. According to their roles and locations, we have devised four components, JEB core, JEB Repeater, JEB Hub, and JEB NS (Name Server). All four components are implemented as Java classes which inherit a common Java interface "JEB". We first describe their main functions as follows.

Routing: Unlike conventional network communication which uses network address for routing, the JEB uses application oriented subject for routing a published message. The subject name is uniquely assigned for a group of publishers and subscribers which exchange messages.

Control and Maintenance: To provide a dynamic communication environment, the JEB supports join and leave protocols for client's participation and publishing and subscribing protocols for subject management. 
Scheduling: To guarantee real-time constraints imposed to publishers and subscribers, the JEB provides priority driven message schedulers in all members of JEB family.

We use a different JEB family module according to the role of the node in global JEB distributed embedded systems. The simplest type of module is JEB Core, which provides JEB facilities for Java clients, either publisher or subscriber, in the same Java virtual machine. When either a publisher or a subscriber needs to connect to other clients located in remote systems, it requires JEB Repeater. JEB Repeater is allowed to have only one communication media, for example Ethernet or VME. With the help of JEB device driver, it provides its clients with JEB publishing and subscribing services. JEB Hub is used for communication between nodes that have different communication media. For example, when a node with a VME interface needs to communicate with a node which has an Ethernet interface, it must be assisted by JEB Hub which supports both communication media. The most sophisticated module of the JEB family is JEB NS, which includes network and subject management functions. JEB NS maintains all publish-subscribe subjects registered in the responsible boundary of the distributed embedded system. The JEB distributed embedded system is configured as a logical hierarchy tree which includes four different types of nodes.

\subsection{JEB Protocol}

In this section, we describe essential JEB protocols such as JEB-boot, join-leave, publish-subscribe.

4.2.1. JEB-boot. The JEB-boot protocol has the responsibility of establishing a connection to a parent node in a logical hierarchy tree. When a JEB node boots, it reports its own configuration information to a parent node. Eventually this information is forwarded to JEB NS. After the network is set up, it will be ready to accept any join and leave requests from publishers and subscribers.

4.2.2. JEB join-leave. JEB allows publishers and subscribers to dynamically join and leave the JEB infrastructure. When a class needs to communicate with JEB facilities, it has to join the JEB infrastructure using the join protocol. By using the join protocol, a class can enroll itself as a member of the JEB infrastructure, and can launch the internal JEB event handler to process future events. When a class needs to depart from the current JEB group, it executes the leave protocol so that all membership related references would be freed.

4.2.3. JEB publish-subscribe. A publisher can send a Java serializable object to interacting subscribers using the publish-subscribe protocol. In JEB, communication between a publisher and a subscriber needs two steps. Firstly, a publisher adds the subject to the subject lists of JEB using jebRegSubject(). Then a subscriber can register itself to that specific subject by jebRegSubscriber(). According to the level with which both are joined, corresponding JEB family members will store routing information (subject, publisher, subscriber). Secondly, when a publisher sends a 
message to JEB, JEB's family will take care of sending the message from the publisher to all registered subscribers in the JEB infrastructure.

\subsection{JEB Classes and API}

The JEB middleware is provided in the form of Java class libraries. In this section, we show one of the most basic classes of JEB middleware. It is a class "JEB" which is used for four members of the JEB family to inherit from. In Table 1, we show the basic methods of a class "JEB".

Table 1. Basic Methods of a Class "JEB"

\begin{tabular}{|l|l|}
\hline int & $\begin{array}{l}\text { jebJoin (String clientName, Object client) } \\
\text { attach a client (publisher or subscriber) to JEB infrastructure }\end{array}$ \\
\hline int & $\begin{array}{l}\text { jebLeave (int clientId, String clientName, Object client) } \\
\text { detach a client (publisher or subscriber) from JEB infrastructure }\end{array}$ \\
\hline int & $\begin{array}{l}\text { JebRegSubject (int clientId, String subjectName) } \\
\text { registers a subject to JEB infrastructure in default mode }\end{array}$ \\
\hline int & $\begin{array}{l}\text { JebRegSubscriber (int clientId, String subjectName) } \\
\text { Registers a subscriber to the subject in the JEB infrastructure. }\end{array}$ \\
\hline int & $\begin{array}{l}\text { JebPublishData (int subjId, Object dataItem) } \\
\text { Publishes dataItem to all subscribers }\end{array}$ \\
\hline Object & $\begin{array}{l}\text { JebFetchData (int subjId) } \\
\text { Fetch the recent Subject Data from JEB }\end{array}$ \\
\hline int & $\begin{array}{l}\text { JebSetClientListener (int subjId, JebClient je) } \\
\text { Register client listener to the subject }\end{array}$ \\
\hline
\end{tabular}

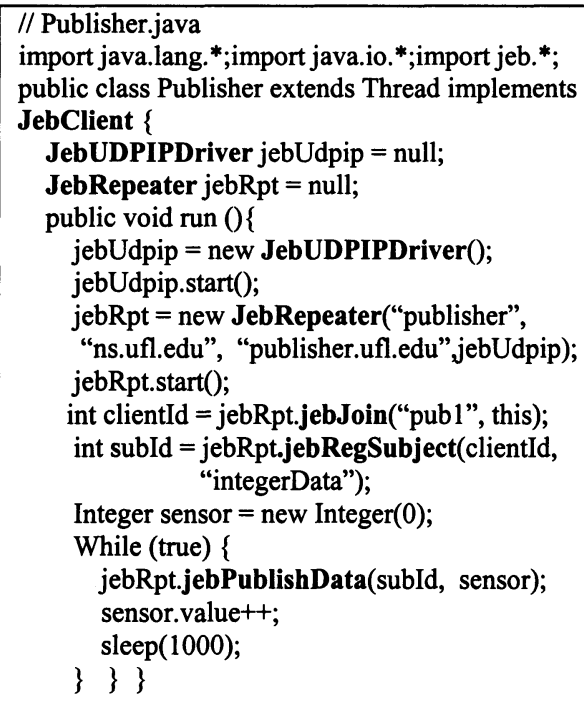

Figure 3. Publisher and Subscriber Sample Program 
In Figure 3, to aid understanding of the middleware, we show two sample programs: a Publisher and a Subscriber. Publisher and subscriber are connected by UDP/IP JEB device driver.

\subsection{JEB Device Driver Model}

The JEB device driver is responsible for connecting JEB middleware and physical communication media. Due to the diversity of physical communication media, we need a middleware independent plug-and-play device driver model. With this concept, it is very easy to change the physical communication media while most of the application code is not touched. All that is required is for application programmers to select the proper JEB device driver and provide configuration information.

4.4.1. JEB Device Driver Architecture. We show the abstract architecture of the JEB device driver model and relationship with the JEB middleware in Figure 4. In the device-driver layer, we use network address for routing messages and bytestreamed Java serialized object for message encoding. In the middleware level, subject and Java object are used correspondingly. The JEB device driver should implement JEB channel management, a channel scheduler, and marshal/unmarshal functions. It should also implement four standard methods of JEB device driver interface (API) as shown in Table 2.

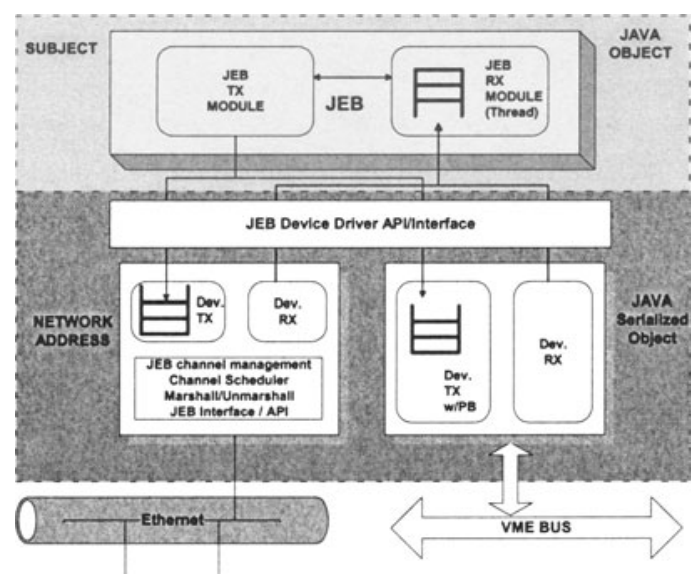

Figure 4. JEB Device Driver Architecture

In the case of message reception, the device driver callbacks the jebOnReceiving method of the JebRXListener interface, which is implemented in every JEB family member. The method stores a received JEB message in the JEB receiving priority queue. In the case of message transmission, the middleware calls the jebDevSend method which stores a JEB message in the transmission-priority queue in the driver. The TX thread will schedule messages and transmit them. 
4.4.2. Device Driver Interface. The only requirement for the JEB device-driver developer is to implement the four interface methods shown in Table 2 . The configuration information can be supplied to the Java constructor of the corresponding device-driver class. For example, in the case of the UDP/IP JEB device driver, JebUDPIPDriver, the constructor is provided with the IP addresses of the local host and a parent host. The port number is fixed for all JEB UDP/IP device drivers. Using the model, we have prototyped two types of JEB device driver for IP/Ethernet and VME Bus. In the case of IP/Ethernet, both TCP and UDP are used for separate device drivers. While the TCP/IP driver can be used for more reliable distributed systems, UDP/IP can be used for less reliable but light-weight system.

Table 2. JEB Device Driver Interface

\begin{tabular}{|l|l|}
\hline public void & $\begin{array}{l}\text { JebDevInit(0 } \\
\text { Initialize JEB Device Driver }\end{array}$ \\
\hline public void & $\begin{array}{l}\text { JebDevStart() } \\
\text { Start JEB Device Driver }\end{array}$ \\
\hline public void & $\begin{array}{l}\text { JebDevSetListener (JebRXListener jrl) } \\
\text { Register RX Message Listener (JEB) }\end{array}$ \\
\hline public void & $\begin{array}{l}\text { JebDevSend(JebMessage jmsg, Vector dest, int priority) } \\
\text { Send a JEB message }\end{array}$ \\
\hline
\end{tabular}

\section{Conclusion}

To provide communication facilities for Java based distributed embedded systems, we have developed Java publish-subscribe middleware. The middleware supports preserving the Java programming style, real time behaviors, variety of communication technologies via the standard device driver model, scalability and interoperability, and exploiting lower level multicast/broadcast. As the Java technology becomes popular in real time systems along with coming real time Java standards, Java publish-subscribe middleware will take an important role in designing complex, evolvable, integrated, and distributed embedded systems in an efficient way.

Our further research area is to support distributed embedded systems which use different languages, such as $\mathrm{Ada}, \mathrm{C}$, and $\mathrm{C}++$. Although there is no doubt that Java will be a popular language in real time applications in the near future, we do not expect that all systems will be written in Java only. So it is important to support different language systems. We consider using either XML or CORBA for exchanging objects between different language systems.

\section{References}

G. Hilderink, J. Broenink, and A. Bakkers (1998) "A new Java Thread Model for Concurrent Programming of Real-time Systems.” Real-Time Magazine. 
G. Pardo-Castellote, S. Schneider, M. Hamilton. (1997) "NDDS: The Real-Tiem PublishSubscribe Network." IEEE workshop on Middleware for Distributed Real-Time Systems and Services.

J-Consortium. (1999) Draft International J Consortium Specification.

L. Carnahan, M. Ruark. (1999) Requirements for Real-time Extensions for the Java Platform, National Institute of Standards and Technology.

M. Colan. (1999) InfoBus 1.2 Specification, Sun Microsystems.

M. Hapner, R. Buffidge, R. Sharma. (1999) Java Message Service Specification, Sun Microsystems.

M. Swick, J. White, and M. Masters. (1998) "A Summary of Communication Middleware Requirements for Advanced Shipboard Computing Systems." IEEE Real-Time Technology and Applications Symposium.

The Real Time for Java Experts Group. (2000), Real Time Specification for Java, Sun Microsystems. 